\title{
Children in today's Japan
}

\begin{abstract}
Shigeru Watanabe* 0283, Japan

\section{Introduction}

For the past decades, the Japanese economy has grown rapidly, starting from the burnt ruins of the war. Its prosperity can be compared with that of the ancient civilizations that left their marks on history. However, the matured society and culture of Japan have now surpassed their capacity and are reaching their limits, the reason for the situation being attributed to the decline of its population. The balance between the birth and death rate in Japan has turned to a minus since 2008, and the country has entered an era of full-blown population decline.
\end{abstract}

Department of Human Development \& Fostering, Division of Pediatric Dentistry, School of Dentistry, Meikai University, 1-1 Keyakidai, Sakado, Saitama 350-

\section{The present situation of children in Japan}

\section{Poverty}

The 2013 UNICEF Innocenti Report Card 11, "Child Well-being in Rich Countries: A comparative overview" [1] reveals that the world that children in Japan now live in is not wholly blessed with light. In comparison with 31 other countries, while Japan is keeping its face as it ranks 1st in the categories "Education" and "Behaviours and risks", it is ranked 16th for "Health and safety" and 21st for "Material well-being (poverty)".

According to the 2013 "Comprehensive Survey of Living Conditions" [2], the poverty line (half of the median of after-tax income) for 2013 was 1.22 million yen (nominal value), and the "relative poverty rate" (household members that do not reach the poverty line) or the percentage of poor families was $16.1 \%$. Furthermore, the "poverty rate for children (under 17 years old)" was $16.3 \%$. This means that 1 out of 6 children under 17 (20 million), and 5 out of 30 children in an elementary or middle school classrooms live in poverty. It is said that in over half of such families, the daily food budget per family member is under 300 yen, and that children in over $80 \%$ of them have malnutrition.

\section{Children's healthcare expenses}

In current Japan, a children's healthcare fee is totally exempt in most regions (the applicable age differs according to prefecture), and because of this, we are prone to think that the disparity due to poverty does not affect the treatment of disease in treatment of children. However, this is not the case. There are municipalities where patients do not have to pay at the hospital counter, and others where patients pay at the counter and later get reimbursed once they submit the receipt. For the latter, patients cannot receive care unless they have the money to pay at the counter.

\section{Neglect}

Since the Child Abuse Prevention Act was adopted in 2000, systems were organized so that organizations fighting child abuse can make joint efforts in order to solve the problem. According to the 2016 announcement (preliminary figures) by the Ministry of Health, Labour and Welfare [3], the number of cases handled at the child consultation center exceeded 0.1 million. The number of children who died from abuse exceeded a total of 1,000 by the year 2014. Thus in terms of numbers and from the dismal realities, child abuse is currently posed as one of the most serious social concerns in Japan [4].

In the past, child abuse existed as "thrashings." It is said that as long as humans form families and live with them, child abuse will continue to exist as part of the "grime of life." If that is the case, measures for eradicating child abuse calls for organization of a thorough framework that reaches individuals and families. However, establishment of preventive measures has been delayed substantially and tragic news on abuse continues to be aired on the news.

\section{Examples of child abuse reported by dentists}

Cases of tooth decay among children are on the decrease as understanding of oral hygiene and day-to-day habits in ordinary families are improving. However, evidence of realities such as lack of interest, disregard, abandonment of childcare, and neglect are appearing in the oral cavities of the children in unexpected forms. Insufficient environment for childcare such as the parent's health issues, divorce, and poverty seem to be disrupting the day-to-day habits, and causing tooth decay.

Figure 1 is an example where an 11-year-old boy was left uncared despite the recommendation after the school health check to treat that tooth decay be treated. This was a single-mother family with two

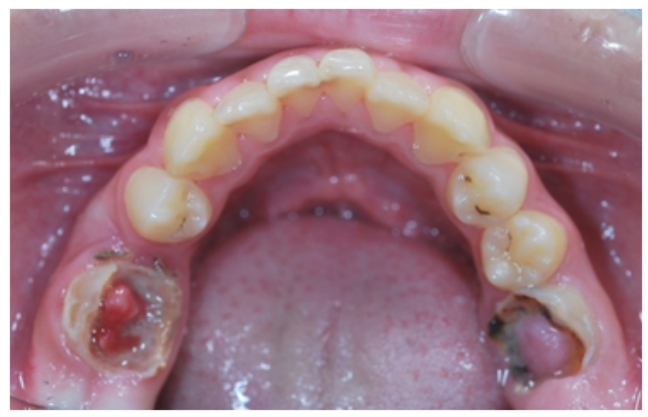

Figure 1. Example where an 11-year-old boy was left uncared despite the recommendation after the school health check to treat that tooth decay be treated.

Correspondence to: Shigeru Watanabe, Department of Human Development \& Fostering, Division of Pediatric Dentistry, School of Dentistry, Meikai University, 1-1 Keyakidai, Sakado, Saitama 350-0283, Japan, Tel: (+81) 49-279-2743, Fax: (+81) 49-279-2743, E-mail: shigeru@dent.meikai.ac.jp

Received: January 08, 2017; Accepted: January 17, 2017; Published: January 20, 2017 
children who were looked after by the grandmother. Mandibular first molars on both sides were affected with chronic proliferative pulpitis. This is a symptom that is rarely seen today, as early treatment became common. The second primary molar can be seen in the vicinity of the left mandibular first molar. Space loss due to tooth decay or tooth loss shows that the teeth have been neglected for a long period of time.

Figure 2 shows the case of a 5 -year -old girl who was neglected after the mother suffered psychological conditions after a divorce. The girl was brought to the hospital by her grandparents. An infected root canal is visible and roots are exposed on several of her anterior teeth. The exposure of the apex of primary tooth is due to neglect of chronic apical periodontitis.

Figure 3 shows a 6 -year-old girl with a significant case of "anterior open bite" caused by thumb sucking. Normally, thumb sucking in children disappears by 3 or 4 with instruction from the guardian, and the open bite is naturally cured. However, in this case the girl was being raised by her grandmother for a long period of time, in a single-mother family.

Decline in the quality of food, continuation of a situation where the disease could not be cured at its early stage, and the inability to receive routine preventive medicine seemed to be the common factors for cases where tooth decay spread because of poverty, even when without malicious neglect [5].

\section{From oral treatment to oral health}

In Japan, the improvement of the national living standards and the spread of interest on oral hygiene had an overall positive effect and children are now almost assured to be free of tooth decay (the average number of decayed teeth for a 12-yr.-old: 1.1. Percentage of 5-year-

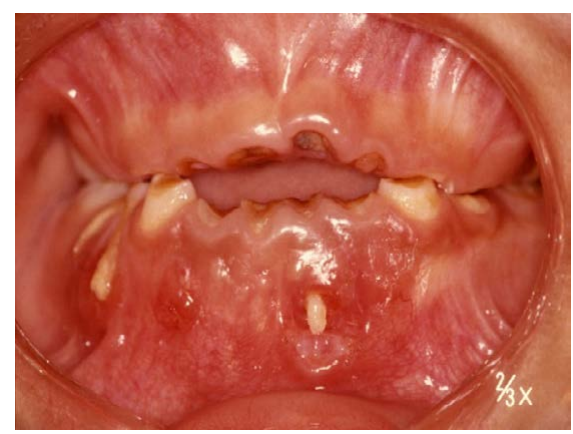

Figure 2. Shows the case of a 5 -year -old girl who was neglected after the mother suffered psychological conditions after a divorce.

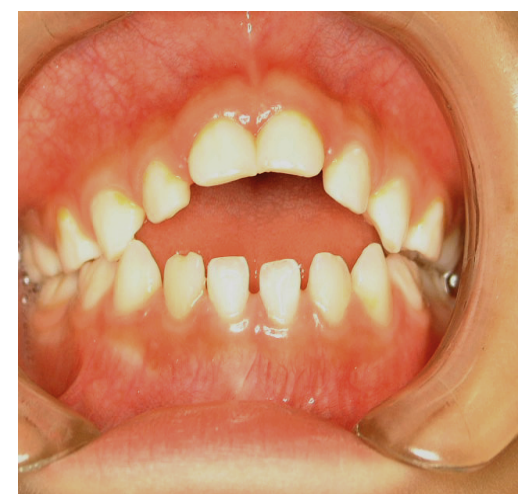

Figure 3. Shows a 6 -year-old girl with a significant case of "anterior open bite" caused by thumb sucking. olds with tooth decay: $42.8 \%$ [5]. Humans are born with an immune system that deals with intrusion of foreign matters. This is true also with the case of tooth decay, and its decline seems to show that the $\mathrm{pH}$ adjustment function of the saliva is starting to work effectively. However, the newly arising problem is that there are still children who are left to live in conditions that surpass this function, children who are forced to live lives beneath our expectations [6]. This problem includes a number of highly social issues, and it is true many of them cannot be solved by dentists. However, it is also true that only dentists can recognize the SOS signs sent by the children's oral cavities. Identifying and supporting children who are forced to live lives grossly below expectation should be recognized as a serious challenge to be undertaken in the field of dentistry.

\section{Conclusion}

The problems due to poverty, such as bullying, suicide, child abuse, insufficient eating habit - these are situations caused by the strains in the society of Japanese children. In such circumstances, specialists on children are being pressed to cooperate with each other to take crosssectional approaches. However, specialists (including dentists) are often too interested in improving their expertise in their own field and they tend only to view the outcomes in the neighboring fields as lessons or examples from which to learn. As a result, the world which in reality is one for the children has been broken down to an aggregate of small fields with different expertise. There are now deep crevasses between the fields, where no one takes heed, and we are seeing reality where we are unable even to point to who is responsible for the children who fall into their darkness.

A world where professionals of different occupations cooperate with each other without walls or crevasses would be the most ideal for those living in it. The challenge for Dentistry now is to take a step outside the specialized field of tooth decay treatment to cooperate with experts from different fields, and to develop as a genre in the field of health sciences involved in diverse issues.

\section{References}

1. Abe S, Takezawa J (2013) UNCEF Innocenti Report Card II. Child well-being in rich countries. A comparing Japan. The Japan Committee for UNICEF, Tokyo.

2. School Health Statistics Survey in 2012. Ministry of education, culture, sports, science and technology (Japan). Available at: http://www.mext.go.jp/b_menu/ toukei/chousa05/hoken/kekka/k_detail/1331751.htm [Accessed January 2017]

3. Overview of the basic survey of the life of the national life in 2013. Ministry of Health, Labor and Welfare (Japan). Available at: http://www.mhlw.go.jp/toukei/ saikin/hw/k-tyosa/k-tyosa12/ [Accessed January 2017]

4. Number of child abuse consultation correspondence at the child consultation center of 2015 (preliminary report). Ministry of Health, Labor and Welfare (Japan) Available at: http://www.mhlw.go.jp/stf/houdou/0000132381.html [Accessed January 2017]

5. Watanabe S, Sato T, Kawasaki F (2014) Oral environment reflects life-Action from dentistry for the Child neglect-. XXth International Congress on Child Abuse and Neglect. Nagoya, Japan.

6. Nakamura A, Komazawa D, Ogihara T, Watanabe S. Children's oral environments and their living conditions: Case Report, Asian Society for Pediatric Research Congress, Nov. 2016, Bangkok.

Copyright: (C)2017 Watanabe S. This is an open-access article distributed under the terms of the Creative Commons Attribution License, which permits unrestricted use, distribution, and reproduction in any medium, provided the original author and source are credited. 